\title{
DESIGN AND PRODUCTION OF HYBRID CIRCUIT ARTWORK
}

\author{
A. D. MILNE \\ Wolfson Microelectronics Institute, The King's Buildings, May field Road, Edinburgh
}

(Received June 11, 1979)

\begin{abstract}
The demand for greater complexity in hybrid circuits requires new techniques to be applied to the production of artwork. The paper discusses the use of a high precision pattern generator and the relevance of integrated circuit CAD sof tware to the production of hybrid circuits.
\end{abstract}

\section{INTRODUCTION}

There is, within the microelectronics industry, a continuing progression towards more complex and, from a functionality point of view, more powerful devices. In the limelight, of course, are silicon integrated circuits with VLSI currently generating considerable interest. However, the sophistication of hybrid circuits has also increased rapidly over the past few years with large numbers of active devices being bonded onto single substrates, very fine interconnection patterns being used and multilayering of conductors becoming a commonly used technique. In order to overcome the demanding design problems and to produce the higher densities of patterns which are now required, new techniques will have to be introduced into the current manufacturing methods. In this paper, an improved method of mask production using a high precision pattern generator will be described. Unlike the pattern generators designed for integrated circuit work, the Wolfson Microelectronics Institute (WMI) machine has been specifically designed to produce large area patterns suitable for hybrid microcircuits and surface acoustic wave (SAW) devices. The use of CAD software to produce pattern generator input will be compared with the direct language input and the problems of automation of the design process will be considered.

\section{HYBRID ARTWORK GENERATION}

The method of artwork generation for hybrid circuits has developed from the taping approach used for printed circuit boards which is adequate for the simplest circuits to the "cut and peel" techniques used to produce artwork at anything from 10 to 100 times the final required size for the more complex patterns. Depending on the magnification either one or two reductions are necessary to produce the final mask. Highly complex patterns can be formed in this way. For very precise patterns however, such as those required for SAW devices, the positional accuracies achievable with this method are inadequate and the areas of the devices are often greater than the field of the reduction lens. The Wolfson Microelectronics Institute pattern generator overcomes these problems by producing the artwork at final size directly on a photographic or photoresist coated plate. By photocomposing the total image from sub-fields, the area of the pattern is only restricted by the size of the plate. This approach is in contrast to the integrated circuit procedure in which a single image is formed at 4 or 10 times the final size which is stepped and repeated to produce a multiple array of small devices.

\section{PATTERN GENERATOR DESIGN}

The major requirements of a pattern generator to produce artwork for hybrid and SAW devices are an ability to produce patterns over large areas with a high degree of absolute accuracy in the positioning of individual features. The artwork for SAW devices, which is perhaps the most demanding of any, requires positional accuracies of sub-micron dimensions over fields of tens of centimetres. 


\subsection{Mechanical Design}

In order to achieve this type of performance, a fairly sophisticated machine is required. The basic concept of the WMI pattern generator is a two axis servo-controlled measuring engine fitted with a light-projection system (Figures 1 and 2). The two carriages, one carrying the photographic place ( $x$-axis) and the other the light projection system ( $y$-axis) are supported by air bearings at the reference flats and guided in two orthogonal directions by an independent system of steering air bearings. The guide rails of the steering air bearings are adjustable to enable fine setting of straightness and orthogonality of both axes independently.

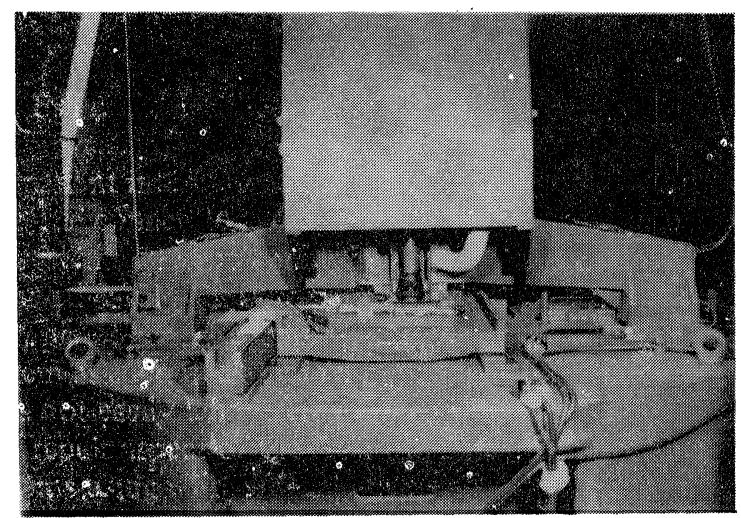

FIGURE 1 Pattern Generator (front).

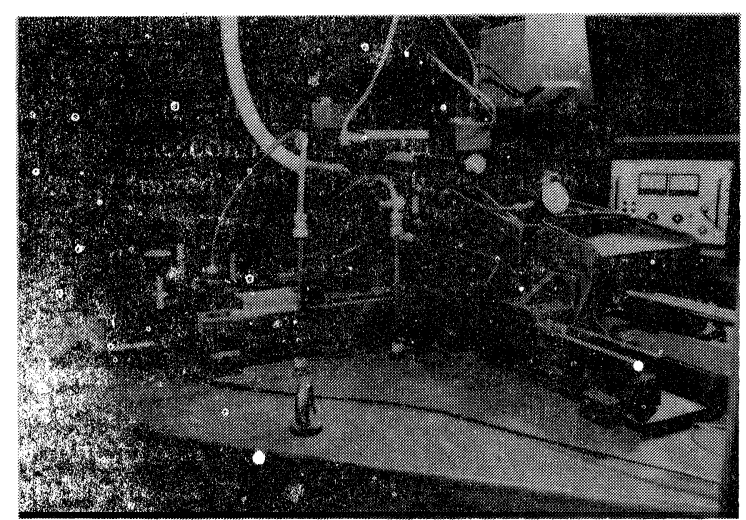

FIGURE 2 Pattern Generator showing drive mechanisms for tables and aperture stage.

The drive system is of course independent of the accuracy of positioning of the carriages which is determined by the moire fringe measurement system. Calibrated reference gratings are mounted on the machine base, parallel to the $x$ and $y$ directions and

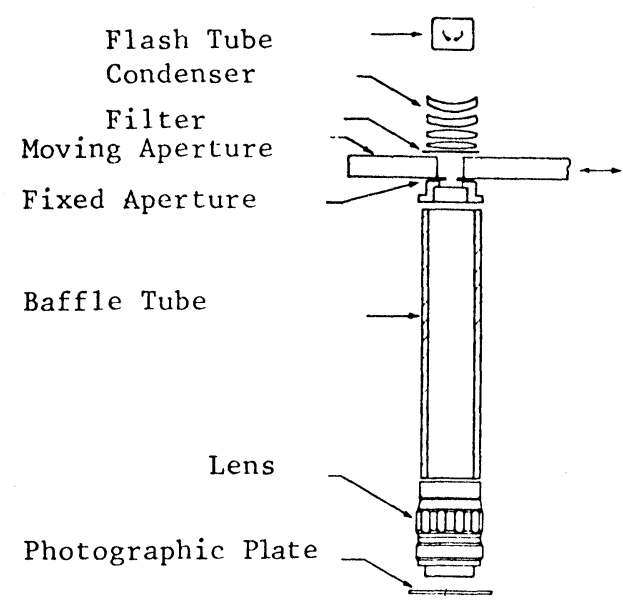

FIGURE 3 Cross section of projection system.

appropriate reading heads are fixed to each moving carriage. The natural pitch of the gratings produces a measurement fringe every $10 \mu \mathrm{m}$, but these fringes are electronically subdivided to give machine step size of $0.5 \mu \mathrm{m}$. In addition to this a linear technique is used to detect the central point of each step, hence enabling the servo system to achieve repeatability not normally expected from purely digital systems. Also, although $0.5 \mu \mathrm{m}$ is the smallest increment available with the $x y$ carriages, patterns can be produced on a finer grid by means of the reticle facilities, as will be described later.

The lighthead uses as its source either a flash tube for exposures on to photographic emulsion or a continuously operating ultraviolet lamp for exposure of photoresists. For simple shapes the image is formed by means of two square apertures aligned parallel to the $x-y$ motion of the machine carriages. One aperture is fixed and the second overlapping aperture is mounted on a stage which can be moved in two directions parallel to the machine axes by

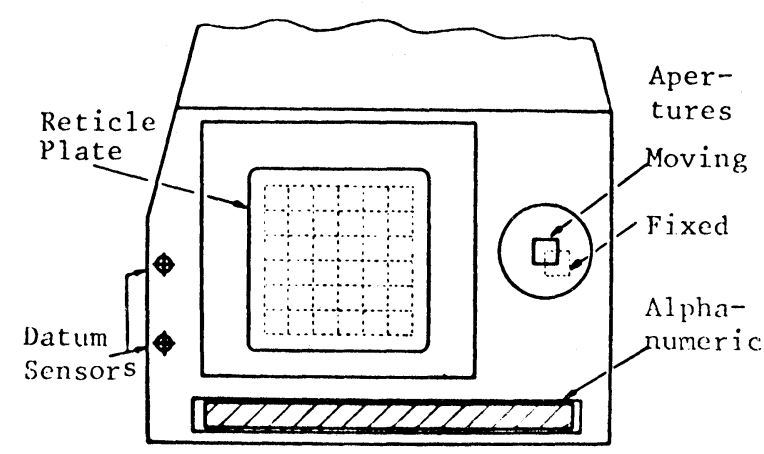

FIGURE 4 Detail of aperture stage. 
stepping motors. Any rectangular aperture from about ten microns to one centimetre square can be produced in steps of five microns. The objective lens, by a $10: 1$ reduction, transforms this to an image variable in size by $0.5 \mu \mathrm{m}$ steps up to a maximum dimension of $1 \mathrm{~mm} \times 1 \mathrm{~mm}$ (Figure 3).

On the same mechanical structure as the moveable aperture is mounted a reticle plate which can contain several predetermined shapes or sub-sections of pattern (Figure 4). The precise alignment of the reticle plate to the machine motions is most important for photocomposition of sub-fields to form a large pattern. Also included on the moveable stage is an alphanumeric reticle which is used for numbering plates and devices.

\subsection{Control}

The pattern generator is controlled by means of a mini-computer which interprets the data input tape and provides the control signals for the machine electronics to position the table, set the aperture or reticle plate and control the light source. The data for mask generation can be produced either with a layout description language or by means of the more sophisticated CAD systems such as the GAELIC ${ }^{1}$ design suite. This suite allows complex interaction with the layout and has certain design rule verification or automation features which are discussed below.

\section{PRODUCING HYBRID ARTWORK}

\subsection{Language Input}

The input commands of the pattern generator contain three elements: the coordinates of the table, the type or size of aperture and whether or not a flash is required. These are defined as $X \Delta Y, S \Delta X \Delta y$ and $F L \Delta n_{f}$ where $n_{f}$ is the number of flashes required. Reticle images can be called by the command $R \Delta n m \Delta p$ which moves the reference point $p$ of the image $n m$ to the position of the optic axis. This is followed immediately by table positioning and flash commands to expose the selected reticle image at the desired point. Certain datum commands are also required and an incremental or absolute coordinate mode has to be selected.

In order to avoid specifying every coordinate of repeated shapes a macro facility is available. With this feature, a group of geometrical shapes is identified and subsequently called by a single command. Up to ten macros, each of 5000 characters, can be defined in any one pattern and they can be nested up to three levels. In order to use a macro in a mask, the call macro command $C M \Delta n_{m} \Delta n_{r}$ is used. This calls macro $n_{m}$ a total of $n_{r}$ times. The input data is obviously much reduced by this feature, particularly for highly regular patterns. In hybrid circuits device bonding pads and even standard resistors could be produced in this way.

Using only the variable aperture to produce rectangles of different sizes is not the most efficient way of producing many patterns and where commonly repeated patterns occur or non-rectilinear geometries are required, the use of the reticle plate is advantageous. The reticle images which are projected through the fixed aperture can be up to $1 \mathrm{~mm}$ square (in final plate dimensions) and 36 such images can be accommodated on a single reticle. The reticle plates themselves can be made by photoreduction of cut and peel artwork or on the machine itself. The former route allows maximum flexibility for producing non-rectilinear patterns such as those required for bubble domain devices but producing the reticle on the machine itself has the important consequence of allowing placement resolution below the basic step size of the machine. Because the reticle images are produced at ten times magnified scale, with a resolution of $0.5 \mu \mathrm{m}$, the position of the pattern in the final artwork can in principle be chosen to the nearest $0.05 \mu \mathrm{m}$.

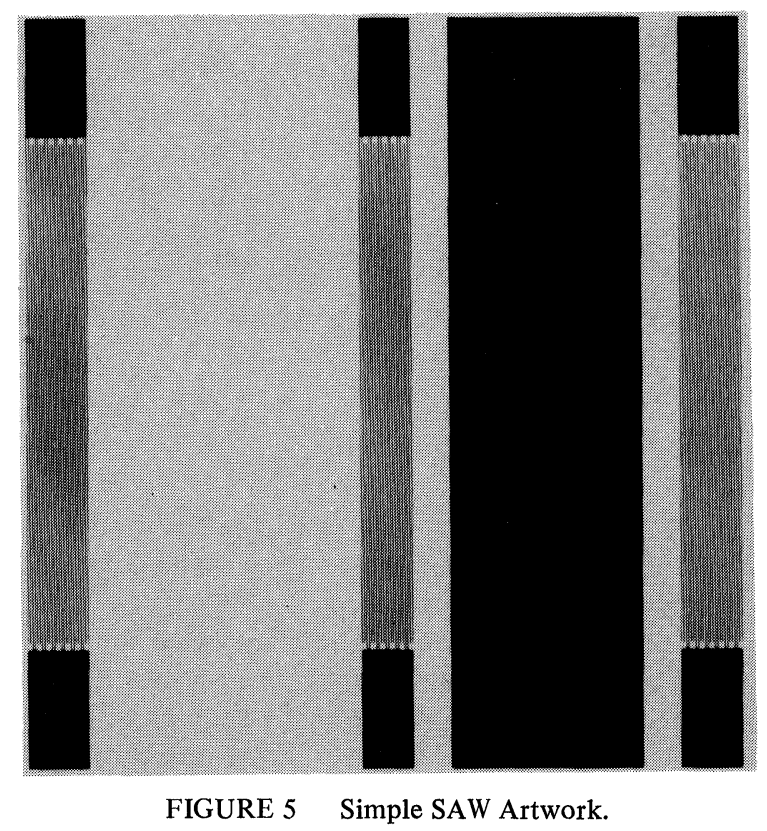


Unfortunately, the overall accuracy of the machine is not sufficient to make this resolution meaningful and a usable figure for the achievable pattern positional precision is $0.2 \mu \mathrm{m}$.

With this simple input language and the sophisticated exposure stage, large and highly complex patterns can be produced over areas up to $300 \times 300 \mathrm{~mm}$. The simple example of a mask produced on the pattern generator shown in Figure 5 is of a SAW device approximately $10 \mathrm{~mm}$ in length. The device was partitioned into seven segments and each segment was drawn as a reticle image, all segments being on the same reticle plate. The individual device pattern was fabricated with 60 exposures and the pattern multiplied in $x$ ( 5 times) and $y$ (6 times) to form a $2^{\prime \prime} \times 2^{\prime \prime}$ mask (Figure 6). Although the sizes of the devices in this example are modest the only inherent limitation in other patterns of greater size or complexity is the 36 reticle images and regular devices several centimetres square have been produced.

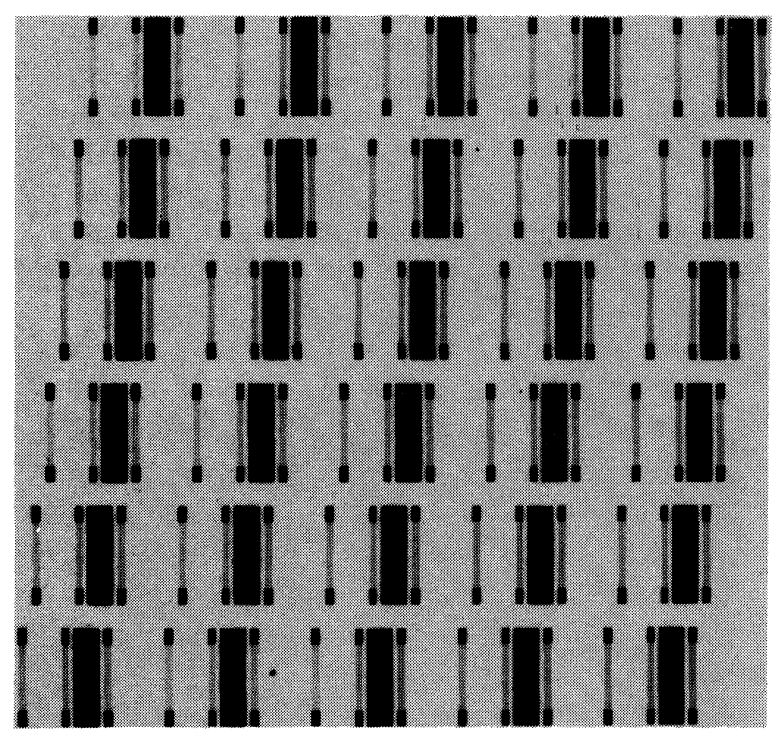

FIGURE 6 Multiple image mask produced at final size without step and repeat.

\subsection{CAD Input}

When the patterns are not so regular and a large number are involved, the use of a CAD system is desirable. The main input to such a system is either by means of a digitiser which requires an accurate drawing of the layout or through a language. The key feature of the system, however, is the ability to interact with data entered in this way to modify it or extend it. A usual approach would be to digitise sections of circuits which could be repeated as exact or modified copies to form a much larger circuit.

Suitably formatted tapes are finally produced to drive the pattern generator. The form of the data on such tapes is normally a simple coordinate string defining the positions of individual rectangles which have to be produced by the pattern generator. This is of a lower level than is ideally suited to the WMI machine as it prevents both the software and hardware macro facilities being exercised.

\subsection{Design Automation}

The introduction of a reasonably powerful computer into the design route does, however, provide the possibility of automating several of the stages. The areas most easily accessible to automation are geometric rule checking and interconnection. In the former the detailed layout rules are a function of the technology and the most efficient way of dealing with this problem is to allow the space relating algorithms access to a library of process rules which can be easily user defined. A program of this type has been constructed by WMI which operates on integrated circuit and hybrid data with equal facility.

The interconnection problem is in principle also common to the technologies of integrated circuits, hybrids and printed circuit boards. However, there are different constraints. With integrated circuits, the number of levels of interconnect is well-defined for a given process and the size of the chip is to some extent elastic. Under these conditions $100 \%$ routing can be achieved with less than a $25 \%$ penalty on area. Printed circuit boards are usually constrained to a fixed dimension and it is desirable to limit the number of interconnect layers to two. This is, of course, a much more restricted situation and it is advantageous to have engineer involvement to optimise the layout and remove conflicts which the software is unable to overcome. The hybrid circuit problem is a combination of the above two technologies. The size of the substrate is most often fixed by packaging constraints but a number of layers of interconnect can be used. Unfortunately there is not a standard hybrid process and the number of layers is not well-defined. Because of the trade-offs between number of layers of interconnect and the use of wire bridges or components to straddle conductors, a degree of manual intervention is highly desirable.

A further area of general automation which is being applied to integrated circuits is function verification based on the layout information. The 
usual procedure is to analyse the layout and identify combinations of shapes as components and compare their connectivity with the original logic. This is satisfactory when functions can be identified by their shapes but as no information can, however, be obtained about the added components of a hybrid, their function would have to be specified in order to make direct comparisons with a logic diagram.

\section{CONCLUSIONS}

The process of hybrid artwork production can be greatly simplified by the utilisation of pattern generators. For small patterns a manual input language is highly desirable but for the more complex layout, sophisticated CAD packages are required. As a result of the development for integrated circuit design, automation techniques are becoming available, some of which are directly applicable to hybrid circuit design. There are special design constraints with hybrid technology however, and special software is required for technology-dependent phases of the design process.

\section{REFERENCE}

1. GAELIC Design Suite, Compeda Ltd., Compeda House, Walkern Road, Stevenage, Herts. 

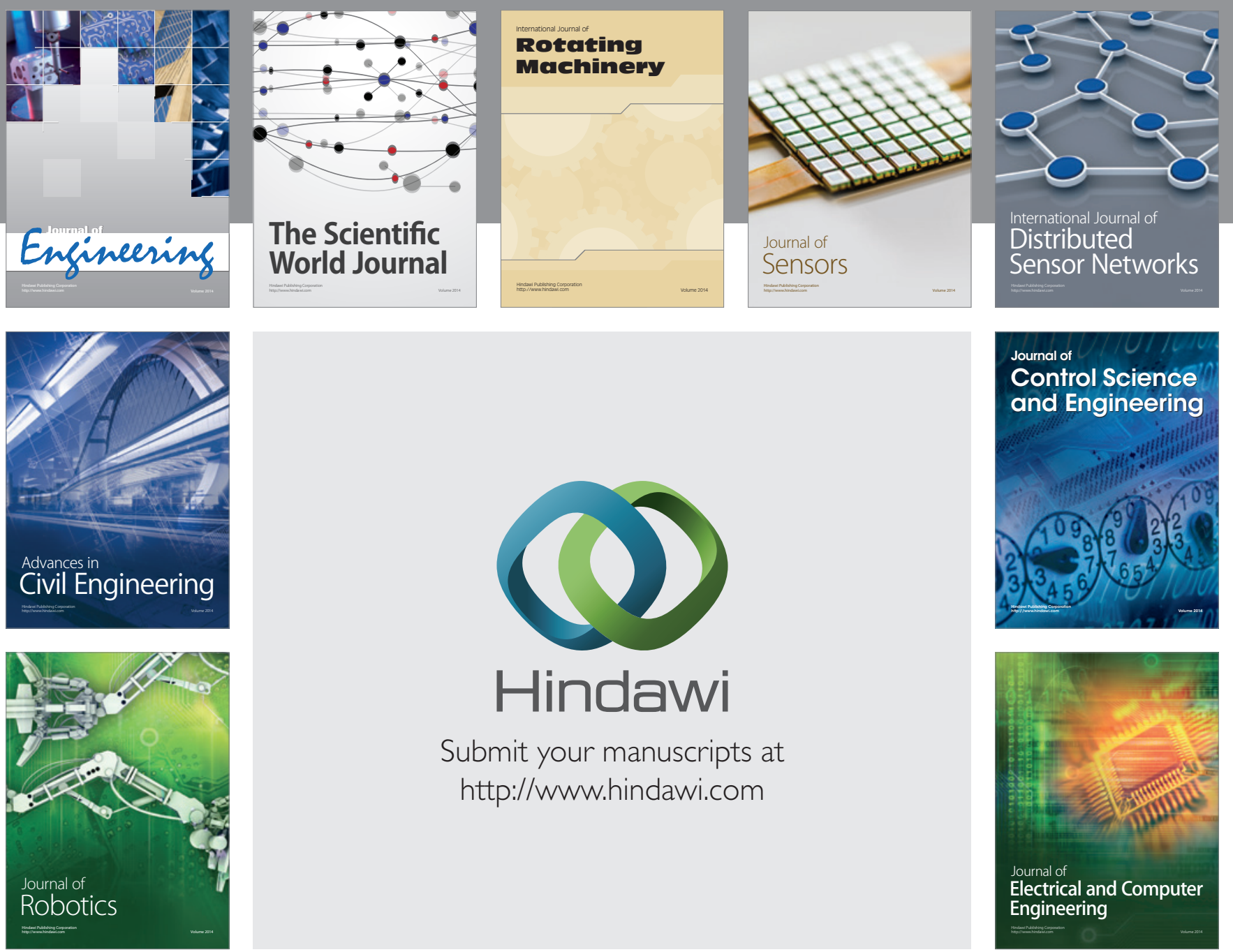

Submit your manuscripts at

http://www.hindawi.com
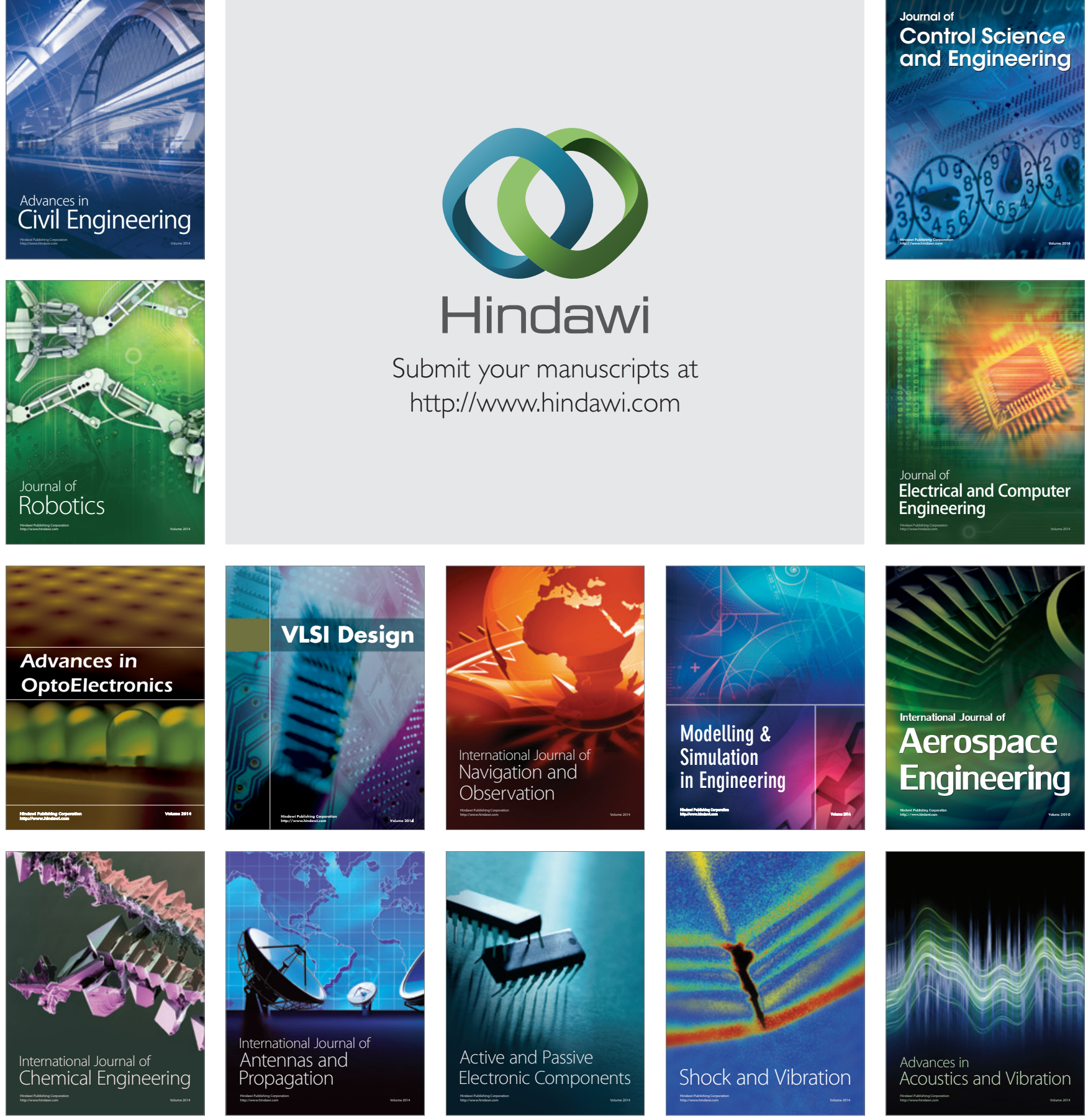